\title{
Avaliação das composições de massas argilosas a partir da incorporação de resíduo da indústria de cerâmica vermelha na obtenção de membranas cerâmicas tubulares
}

\author{
Evaluation of clayey masses compositions \\ starting from the residue incorporation \\ of the red ceramic industry to obtain tubular \\ ceramic membranes
}

Adriano Lima da Silva ${ }^{1}$, Alexsandra Cristina Chaves ${ }^{1}$, Carlos Bruno Barreto Luna ${ }^{1}$,Gelmires de Araújo Neves ${ }^{1}$, Hélio de Lucena Lira ${ }^{1}$

\footnotetext{
${ }^{1}$ Unidade Acadêmica de Engenharia de Materiais, UAEMa/CCT/UFCG, Rua: Aprígio Veloso, 882, Cep: 58429-140, Campina Grande-PB, e-mail: adrianolimadasilva@hotmail.com; alexsandra.chaves@ifap.edu.br; brunobarretodemaufcg@hotmail.com; gelmires@ufcg.edu.br; helio@ufcg.edu.br.
}

\section{RESUMO}

O descarte inapropriado de resíduos das indústrias da cerâmica vermelha é bastante elevado. Um dos maiores desafios da atualidade é a investigação de processos para a obtenção de materiais alternativos, que permitam o emprego destes resíduos na fabricação de novos materiais. Este trabalho tem como objetivo estudar composições de massas argilosas a partir da incorporação de resíduo da indústria de cerâmica vermelha para uso em membranas cerâmicas tubulares. Foram estabelecidas duas composições de massa cerâmica; composição A (50\% de resíduo) e composição B (70\% de resíduo). As análises granulométricas das massas cerâmicas apresentaram um tamanho médio de partículas que prever membranas na escala de microfiltração. Outro fator observado está relacionado ao aumento da quantidade de resíduo, o mesmo, favoreceu a diminuição da plasticidade na massa cerâmica. Observou-se também um aumento na porosidade aparente, provavelmente pelo possível crescimento do número de poros maiores, decorrentes da temperatura elevada de sinterização e do próprio aumento da quantidade de resíduo.

Palavras-chave: Resíduos, tijolos, reaproveitamento, membranas cerâmicas.

\section{ABSTRACT}

The inappropriate residue disposal of red ceramic industry is very high. Nowadays, one of the major challenges is the investigation of processes to obtain alternative materials, enabling the use of these residues to manufacture new materials. This work's objective is to study clayey masses' compositions starting from the residue incorporation of the red ceramic industry to be used in tubular ceramic membranes. Two compositions of ceramic masses were established, composition A (50\% of residue) and composition B ( $70 \%$ of residue). Granulometric analysis of the ceramic masses presented an average size of particles, what indicates membranes in the microfiltration scale. Another observed factor is related to the increase of residue amount, what favored a decrease in the ceramic mass' plasticity. A rise in the apparent porosity was also observed, probably because of a possible growing in the bigger pores numbers, due to the sintering high temperature and the elevation of residue quantity itself.

Keywords: Waste, bricks, reutilization, ceramic membranes.

\section{INTRODUÇÃO}

Nos últimos anos, a pesquisa sobre o reaproveitamento dos resíduos industriais e de construção vem sendo intensificada em todo o mundo. O reaproveitamento dos resíduos urbanos ganha evidência, em virtude do crescente volume de rejeitos sólidos e da indisponibilidade cada vez maior de locais para descarte desse material, em particular em grandes centros urbanos, com elevada densidade demográfica. Sabe-se que os rejeitos urbanos agridem o meio ambiente, em virtude da falta de tratamento adequado [1]. Segundo MENEZES et 
al. [2] os resíduos das indústrias e da construção civil tem uma contribuição importante para esta questão, representando um grave problema ambiental e econômico.

As principais fontes de resíduos derivados de materiais argilosos vêm da indústria cerâmica, da construção civil e da demolição de edifícios. Nas indústrias de cerâmica, uma parte significativa das perdas na fabricação de blocos e telhas não é retornada ao processo de produção. Na construção de edifícios, o resíduo é produzido nas suas diversas etapas e estes são depositados em aterros, sem qualquer separação ou reaproveitamento. Já a demolição de edifícios muitas vezes não é seletiva, tornando o reaproveitamento do resíduo cerâmico economicamente inviável [3]. Portanto, baseada na problemática do despejo em grande quantidade de resíduo proveniente da indústria cerâmica, da construção civil e demolição, tem-se a carência de pesquisas sobre a aplicação destes rejeitos industriais. Sabe-se que, geralmente, a indústria de cerâmicas vermelha produz uma quantidade bastante elevada de resíduos sólidos, que começam a ser acumulados desde o armazenamento nas alvenarias em decorrência dos produtos quebrados devido à má manipulação [4]. Sendo assim, sabe-se que os resíduos da indústria cerâmica é uma fonte de agressão ambiental e desperdício de matériaprima. Estes materiais apresentam resistência relativamente alta a agentes biológicos e às intempéries, causando assim sérios problemas à comunidade em geral quando descartados. Logo, tanto os aspectos econômicos, quanto a poluição ambiental são justificativas para pesquisas no sentido de promover a reciclagem destes materiais.

Uma das tecnologias com potencial do reaproveitamento dos resíduos de cerâmica é através do desenvolvimento de membranas [5]. As membranas são amplamente empregadas em processos de separação nas áreas de tratamento de rejeitos industriais, processamento de alimentos, dessalinização de águas, processos biomédicos, têxteis e químicos. Atualmente, membranas cerâmicas de microfiltração e ultrafiltração estão disponíveis comercialmente e são aplicadas nas áreas de alimentos, bebidas e biotecnologia, representando cerca de $12 \%$ das membranas utilizadas nestas aplicações [6].

Poucos trabalhos têm sido publicados sobre membranas cerâmicas a base de resíduos. Na pesquisa de LIMA et al. [7] foi desenvolvido membranas cerâmicas tubulares de baixo custo utilizando resíduo de granito. Inicialmente o resíduo granítico foi caracterizado através de análise granulométrica, análise química por fluorescência de raios X, e difração de raios X. As membranas produzidas com argila bentonita (35\%), quartzo (35\%) e resíduo de granito (30\%) foram sinterizadas a temperaturas de $750,800,850$ e $900^{\circ} \mathrm{C}$ e caracterizadas por micrografia eletrônica de varredura, porosimetria por intrusão de mercúrio e análise de fluxo tangencial com água deionizada. Os resultados mostraram que o resíduo de granito apresentou média distribuição de tamanho de partículas, com diâmetro médio dos grãos de 18,46 $\mu \mathrm{m}$ e elevados teores de sílica e alumina. As membranas apresentaram diâmetro médio dos poros na faixa entre micro e ultrafiltração e fluxo tangencial entre 10,0 e $20,0 \mathrm{~L} / \mathrm{h} \cdot \mathrm{m}^{2}$.

O objetivo deste trabalho é o desenvolvimento de membranas cerâmicas de baixo custo a partir do aproveitamento de resíduo da indústria de cerâmica vermelha.

\section{MATERIAIS E MÉTODOS}

As matérias-primas utilizadas foram: argila bentonita proveniente da região de Cubatí (PB), resíduos de tijolos cerâmicos vermelhos cedidos pela CIMAC (empresa de materiais de construção localizada em Campina Grande - PB), água e oleína.

Os resíduos inicialmente foram submetidos a um processo de beneficiamento através de moagem em moinho de bolas e, posteriormente, passado em peneira ABNT No $200(0,074 \mathrm{~mm})$, objetivando o alcance de um pó cerâmico cuja granulometria inferior a $74 \mu \mathrm{m}$. Após o beneficiamento foram estabelecidas as composições das massas cerâmicas, conforme Tabela 1.

Tabela 1: Composições das massas cerâmicas.

\begin{tabular}{|c|c|c|}
\hline Amostras & $\begin{array}{c}\text { Porcentagem } \\
\text { Resíduo - Bentonita }\end{array}$ & $\begin{array}{c}\text { Massa para } 2000 \mathrm{~g} \text { de } \\
\text { massa total } \\
\text { Resíduo - Bentonita }\end{array}$ \\
\hline Composição 50 \% de resíduo & $50 \% \quad 50 \%$ & $1000 \mathrm{~g} \quad 1000 \mathrm{~g}$ \\
\hline Composição 70 \% de resíduo & $70 \%$ & $1400 \mathrm{~g}$ \\
\hline
\end{tabular}

A caracterização das composições foi efetuada por meio das seguintes técnicas: limites de Atenberg (LL,LP,IP) norma da ABNT NBR-6459 [8] e NBR-7180/1994 [9], análise granulométrica por difração de laser (AG) (equipamento Cilas, Modelo 1064 L/D), análise química por fluorescência de raios X (EDX 720 Shimadzu), análise termogravimétrica (ATG) e análise térmica diferencial (ATD) (sistema simultâneo de análises térmicas TA $60 \mathrm{H}$ Shimadzu), com taxa de aquecimento $12,5^{\circ} \mathrm{C} / \mathrm{min}$ sob atmosfera de ar (a temperatura máxima para ambos os casos foi $1000^{\circ} \mathrm{C}$ e o padrão utilizado na ATD foi óxido de alumínio $\left(\mathrm{Al}_{2} \mathrm{O}_{3}\right)$ cal- 
cinado), e difração de raios X (DRX) (XRD6000 Shimadzu), com radiação Cuka (40 kV/30 mA) e velocidade do goniômetro de $2^{\circ} / \mathrm{min}$ com passo de $0,02^{\circ}$, na faixa de $2^{\circ}$ a $60^{\circ}$.

Para a conformação das membranas cerâmicas, a massa foi envolvida com $3 \mathrm{ml}$ de oleína e $400 \mathrm{ml}$ de água a partir da homogeneização mecânica através de um misturador planetário de bancada Palivest modelo 13010-359. As membranas em formato tubular foram obtidas por extrusão, utilizando-se uma extrusora a vácuo, fabricada pela Verdés modelo 051. Em que as membranas tubulares apresentam os diâmetros do molde acoplado a extrusora, viabilizando assim as membranas ao ensaio de fluxo em escala laboratorial, a qual a universidade dispõe. As peças obtidas foram submetidas a duas etapas de secagem. A primeira etapa de secagem natural durante cinco dias, cobertas com pano úmido e, a segunda etapa, na estufa com temperatura controlada em torno de $60^{\circ} \mathrm{C}$ por $48 \mathrm{~h}$. Passada essa etapa, as peças cerâmicas foram cortadas com comprimento aproximado de $80 \mathrm{~mm}$, posteriormente sinterizadas no forno elétrico Maitec à temperatura de $800^{\circ} \mathrm{C}$. Após sinterização, as membranas cerâmicas foram submetidas ao ensaio de microscopia eletrônica de varredura (MEV) marca Philips, Jeol LV 5600, operando-se em $15 \mathrm{kV}$ e contraste de elétrons retroespalhados para análises das amostras, teste de porosidade (pelo princípio de Arquimedes), e ao ensaio de fluxo em escala de laboratório.

\section{RESULTADOS E DISCUSSÃO}

\subsection{Ensaio de Plasticidade}

Na Tabela 2 estão ilustrados os resultados dos limites de liquidez (LL), plasticidade (LP) e índice de plasticidades (IP) das composições das massas estudadas.

Tabela 2: Limites de Liquidez (LL), Plasticidades (LP) e Índice de Plasticidade (IP) dos Compostos com 50\% e 70\% de resíduo.

\begin{tabular}{c|c|c|c}
\hline Amostra & LL (\%) & LP (\%) & IP (\%) \\
\hline $\begin{array}{c}\text { Composição com 50 \% } \\
\text { de resíduo (A) }\end{array}$ & 59,0 & 30,0 & 28,1 \\
\hline $\begin{array}{c}\text { Composição com 70 \% } \\
\text { de resíduo (B) }\end{array}$ & 47,0 & 29,7 & 17,3 \\
\hline
\end{tabular}

Analisando os valores apresentados na Tabela 2, observa-se que os limites de liquidez (LL) e de plasticidade (LP) foram de 59 e $30 \%$, e de 47 e $29,7 \%$, respectivamente para as amostras A e B, enquanto o índice de plasticidade foi de 28,1 e $17,3 \%$. Com base em dados da literatura [10] verifica-se que os valores obtidos estão dentro dos intervalos observados para cerâmica vermelha, 30 a 60\% para o LL, 15 a 30\% para o LP e 10 a $30 \%$ para o IP. Ainda constata-se que o limite de plasticidade (LP), encontra-se na faixa de valores encontrados para massas cerâmicas conformadas pelo processo de extrusão. Em relação aos valores do índice de plasticidade (IP), observa-se que o valor, expresso na tabela 2 permitiu concluir, que segundo critérios utilizados em mecânica dos solos, a massa cerâmica é classificada como Plástica [11].

\subsection{Ensaio de Granulometria a Laser}

A Figura 1 apresenta a distribuição granulométrica das partículas das composições A e B. Analisando-se as curvas da Figura 1, verifica-se que a distribuição de tamanho de partículas das composições apresentaram $25 \%$ da fração argila $(\mathrm{D}<2 \mu \mathrm{m}), 65 \%$ da fração silte $(2 \mu \mathrm{m}<\mathrm{D}<20 \mu \mathrm{m})$ e $10 \%$ da fração areia $(\mathrm{D}>20 \mu \mathrm{m})$.

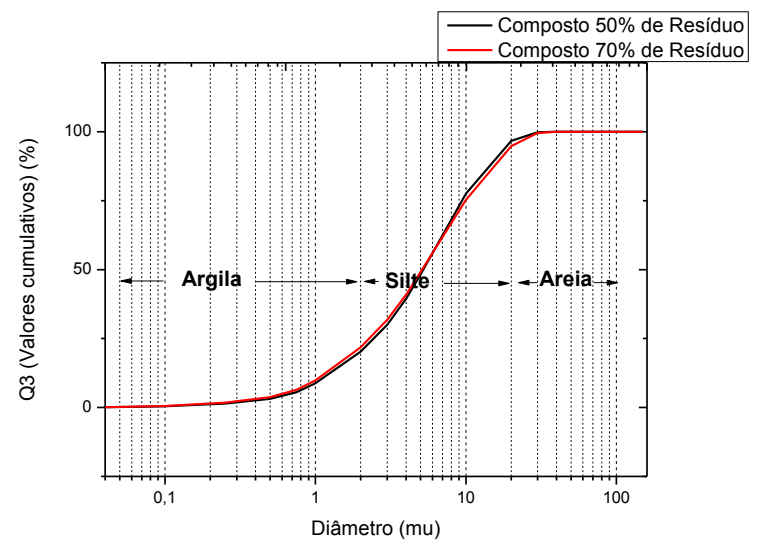

Figura 1: Distribuição granulométrica das composições 50\% e 70\% de resíduo respectivamente. 
A Tabela 3 representa a massa acumulada dos compostos, onde $\mathrm{D}_{10}, \mathrm{D}_{50}$ e $\mathrm{D}_{90}$, são porcentagens $(10 \%$, $50 \%$ e $90 \%$ ) nos quais o diâmetro das partículas se encontra abaixo do especificado.

Tabela 3: Composição granulométrica da massa cerâmica nas composições $50 \%$ e $70 \%$ de resíduo.

\begin{tabular}{c|c|c|c|c}
\hline Amostra & $\mathbf{D}_{10}(\boldsymbol{\mu m})$ & $\mathbf{D}_{50}(\boldsymbol{\mu m})$ & $\mathbf{D}_{90}(\boldsymbol{\mu m})$ & $\mathbf{D}_{\text {médio }}(\boldsymbol{\mu m})$ \\
\hline $\begin{array}{c}\text { Composição } 50 \% \text { de } \\
\text { resíduo }\end{array}$ & 1,10 & 5,21 & 14,63 & 6,72 \\
\hline $\begin{array}{c}\text { Composição } 70 \% \text { de } \\
\text { resíduo }\end{array}$ & 1,01 & 5,11 & 16,19 & 7,04 \\
\hline
\end{tabular}

Segundo os dados apresentados na Tabela 3, o diâmetro médio das partículas do composto com $50 \%$ de resíduo é $6,72 \mu \mathrm{m}$ e do composto com $70 \%$ de resíduo é 7,04 $\mu \mathrm{m}$. Conforme CHAVES et al. [12] embora esta distribuição granulométrica seja bastante larga, verifica-se um alto teor de partículas com tamanhos na faixa de 2 a $10 \mu \mathrm{m}$, bem como uma fração considerável de partículas abaixo de $2 \mu \mathrm{m}$. É de se esperar com esta distribuição uma boa compactação entre partículas finas e grossas após conformação, de maneira que se tenham poros submicrométricos e um elevado grau de sinterização após queima.

\subsection{Análise Química (EDX)}

$\mathrm{Na}$ Tabela 4 estão apresentadas as composições químicas das massas cerâmicas com 50 e $70 \%$ de resíduo.

Tabela 4: Composições químicas das massas com $50 \%$ e $70 \%$ de resíduo respectivamente.

\begin{tabular}{c|c|c}
\hline Substância & $\begin{array}{c}\text { Composição 50 \% de } \\
\text { resíduo }\end{array}$ & $\begin{array}{c}\text { Composição 70 \% de } \\
\text { resíduo }\end{array}$ \\
\hline $\mathrm{SiO}_{2}$ & 55,845 & 55,719 \\
\hline $\mathrm{Al}_{2} \mathrm{O}_{3}$ & 28,953 & 27,382 \\
\hline $\mathrm{Fe}_{2} \mathrm{O}_{3}$ & 7,728 & 9,138 \\
\hline $\mathrm{MgO}$ & 2,619 & 2,591 \\
\hline $\mathrm{K}_{2} \mathrm{O}$ & 1,432 & 1,807 \\
\hline $\mathrm{TiO}_{2}$ & 1,572 & 1,668 \\
\hline $\mathrm{CaO}$ & 1,585 & 1,504 \\
\hline $\mathrm{Outros}$ & 0,264 & 0,190 \\
\hline
\end{tabular}

Com base nos dados da Tabela 4, verifica-se que as massas cerâmicas apresentam composição química com teores de $\mathrm{SiO}_{2}$ e $\mathrm{Al}_{2} \mathrm{O}_{3}$, típica de massas plásticas para cerâmica vermelha. A presença de óxido de ferro $\left(\mathrm{Fe}_{2} \mathrm{O}_{3}\right)$ com cerca de $9 \%$ na composição com $70 \%$ de resíduo, e com aproximadamente $7,8 \%$ na composição com $50 \%$ de resíduo confere a cor vermelha após queima. Observa-se ainda, que as duas amostras apresentaram baixos teores de $\mathrm{CaO}$ e $\mathrm{MgO}$, sendo que os mesmos são importantes, pois atuam como fundentes. Esses óxidos em elevadas quantidades poderia interferir no processo de sinterização, favorecendo o surgimento de formação de fase liquida e o provável entupimento de poros.

\subsection{Análise Termogravimétrica (TG)}

A Figura 2 ilustra as curvas termogravimétricas das duas composições e a Tabela 5 os intervalos de decomposição extraídas do gráfico.

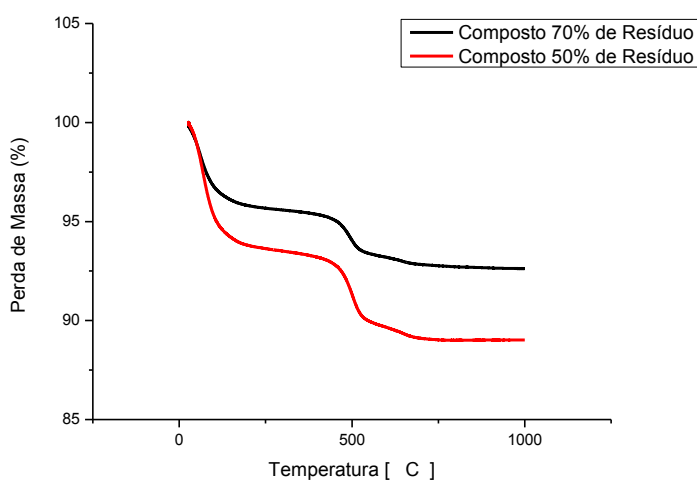

Figura 2: Curvas termogravimétricas das composições com $70 \%$ e $50 \%$ de resíduo. 
Tabela 5: Decomposições térmicas dos compostos A e B.

\begin{tabular}{|c|c|c|c|c|}
\hline \multirow{2}{*}{ Amostra } & \multirow[b]{2}{*}{ Etapas } & \multirow{2}{*}{$\begin{array}{c}\text { Variação de } \\
\text { Temperatura } \\
\left({ }^{\circ} \mathrm{C}\right)\end{array}$} & \multicolumn{2}{|c|}{ Perda de Massa } \\
\hline & & & $(\%)$ & (mg) \\
\hline \multirow{3}{*}{$\begin{array}{c}\text { Composição } 50 \% \\
\text { de resíduo }\end{array}$} & 1 & $26,53-238,23$ & 6,337 & 1,694 \\
\hline & 2 & $238,23-651,05$ & 4,351 & 1,163 \\
\hline & 3 & $651,05-1000,0$ & 0,303 & 0,081 \\
\hline \multirow{3}{*}{$\begin{array}{c}\text { Composição } 70 \% \\
\text { de resíduo }\end{array}$} & 1 & $28,56-258,60$ & 4,128 & 1,252 \\
\hline & 2 & $258,60-601,89$ & 2,466 & 0,748 \\
\hline & 3 & $601,89-1000,0$ & 0,564 & 0,171 \\
\hline
\end{tabular}

Observa-se que há três estágios de perda de massa para as duas composições. O primeiro, possivelmente relacionado à eliminação de água adsorvida e estrutural do material, havendo nesta etapa a maior perda de massa durante o processo total. No segundo estágio de perda, relacionado à perda de matéria orgânica e hidroxilas presentes na composição e, finalmente, o último estágio de perda, que pode esta associada com o desenvolvimento de núcleos de mulita ou uma fase com estrutura de espinélio [13,14].

\subsection{Difração de Raios-X (DRX)}

A Figura 3 apresenta os difratogramas dos resíduos A e B, no qual se verificou a presença das seguintes fases mineralógicas: quartzo, caulinita, esmectita e moscovita, sendo o pico mais intenso de ambos os gráficos para o de quartzo, identificados por meio das distâncias interplanares basais de cada pico. Os resultados de ambos os resíduos está de acordo com a composição da massa cerâmica obtida a partir da mistura do resíduo de tijolo com argila bentonita, e sua composição ser típica de uma cerâmica vermelha.

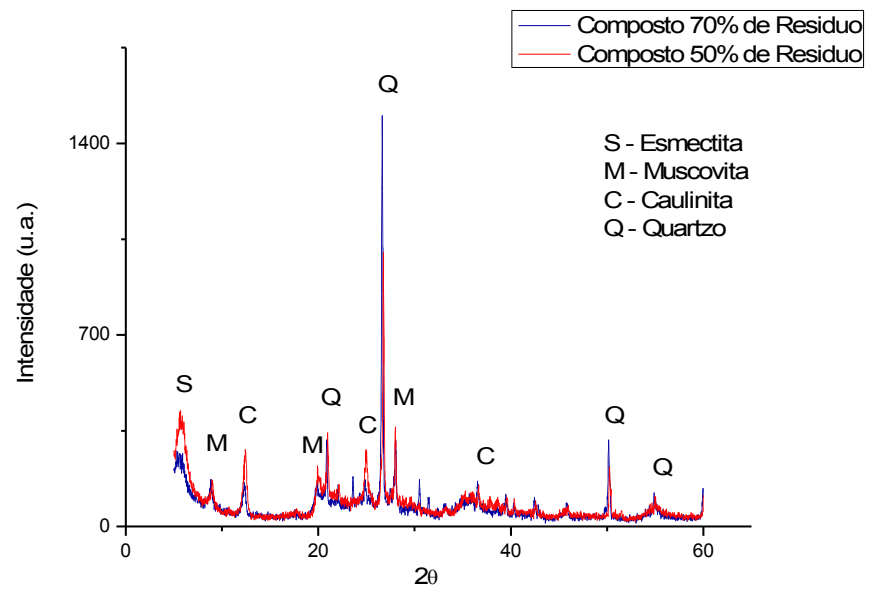

Figura 3: Difratograma de raios- $X$ das massas cerâmicas.

\subsection{Caracterização Morfológica (MEV)}

As Figuras 4 e 5 apresentam as micrografias eletrônicas das membranas cerâmicas desenvolvidas com 50 e $70 \%$ de resíduo respectivamente. 


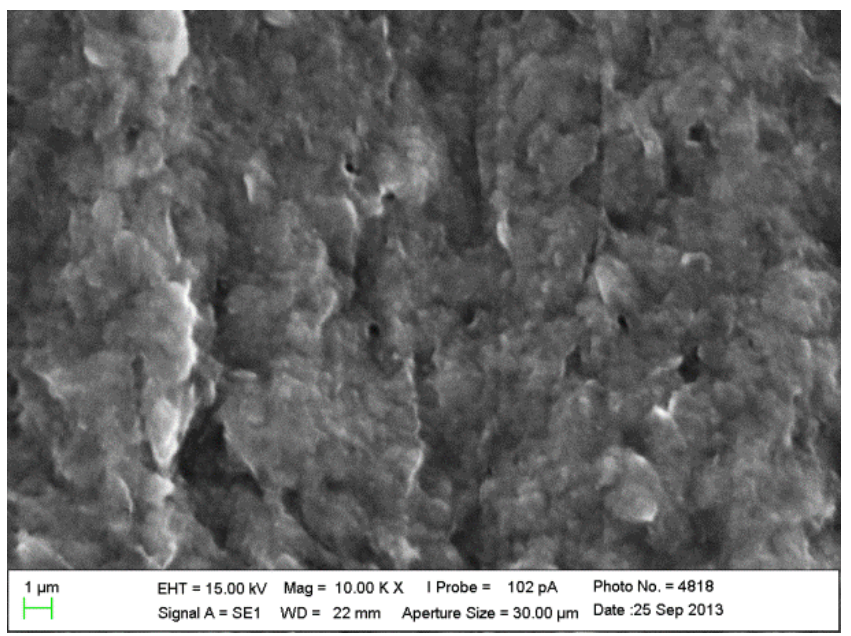

Figura 4: Micrografia da membrana composta com $50 \%$ de resíduo sinterizada a $800^{\circ} \mathrm{C}$, com ampliação de $1000 \mathrm{x}$.

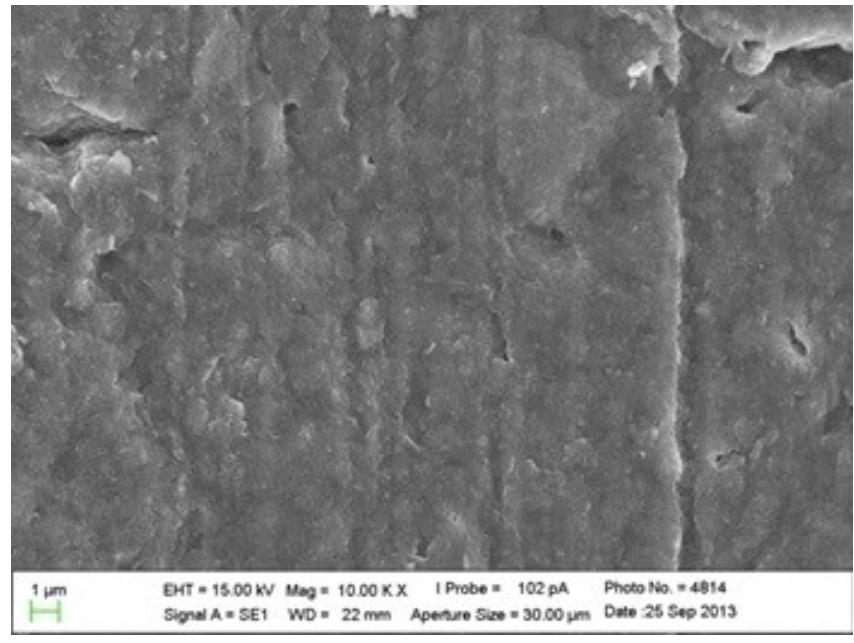

Figura 5: Micrografia da membrana composta com $70 \%$ de resíduo sinterizada a $800^{\circ} \mathrm{C}$, com ampliação de $1000 \mathrm{x}$.

Analisando as Figuras 4 e 5 não foi possível estimar com precisão o tamanho dos poros em nenhum dos casos, em virtude de não haver uniformidade na distribuição. É possível verificar ausência de trincas e defeitos superficiais nas membranas. Ainda constata-se a presença de poros nas seções transversais, todavia as micrografias não permitem, porém, estimar com precisão o tamanho dos poros.

\subsection{Porosidade Aparente pelo Principio de Arquimedes}

$\mathrm{Na}$ Tabela 6 estão contidos os valores da porosidade aparente das composições estudadas.

Tabela 6: Porosidade das membranas investigadas.

\begin{tabular}{c|c}
\hline Amostra & Porosidade Aparente $(\varepsilon \mathbf{A})$ \\
\hline Composição $50 \%$ de resíduo & $31,03 \%$ \\
\hline Composição $70 \%$ de resíduo & $32,14 \%$ \\
\hline
\end{tabular}

Observando os valores na Tabela 6 , verifica-se que a composição com $70 \%$ de resíduo apresentou uma porosidade levemente superior à composição com $50 \%$ de resíduo, o que pode está relacionado à presença de poros maiores nesta composição. É importante salientar que a porosidade determinada pelo princípio de Arquimedes considera apenas os poros abertos. 


\subsection{Ensaio de Fluxo nas Membranas}

Na Figura 6 estão apresentados os resultados do fluxo permeados das membranas das composições $50 \%$ e $70 \%$ de resíduo respectivamente.

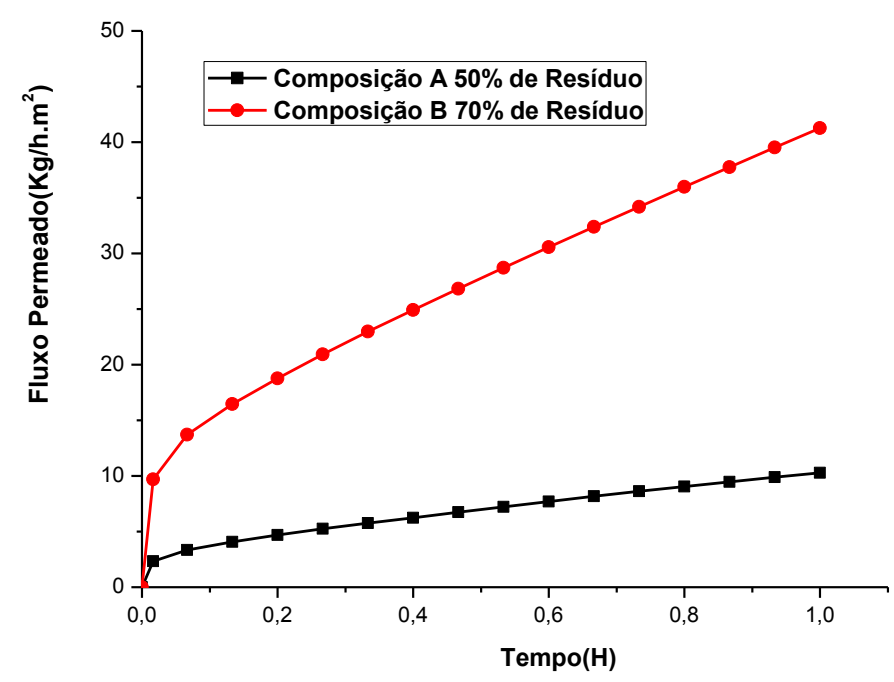

Figura 6: Fluxo permeado das composições $50 \%$ e $70 \%$ de resíduo a $800{ }^{\circ} \mathrm{C}$.

Analisando a Figura 6, foi possível observar que a composição B com 70\% de resíduo obteve maior volume de fluxo permeado do que a composição A com $50 \%$ de resíduo. Esse fato pode está relacionado a um aumento da porosidade da membrana. Para ambas, houve inicialmente um aumento do fluxo permeado, porém com o decorrer do tempo do ensaio as duas curvas vão tendendo a atingir os seus valores constantes, que é tomado como referência para a membrana $[15,16]$.

\section{CONCLUSÕES}

Com o estudo realizado foi possível verificar que membranas cerâmicas tubulares produzidas a partir da incorporação de resíduos de tijolos cerâmicos, foram realizadas com sucesso para teores de $50 \%$ e $70 \%$ de resíduo em peso, onde a membrana que permeou um maior fluxo foi com a incorporação de $70 \%$ de resíduo. Os resultados de caracterização indicaram que a massa cerâmica pode ser utilizada como matéria-prima promissora, para obtenção de membranas cerâmicas na possível escala de microfiltração.

A facilidade de obtenção, de preparo e o baixo custo desse material, justifica a produção da membrana à base de resíduos que atingiu diâmetro de poro e porosidade voltada para aplicações nos variados processos de microfiltração, viabilizando, dessa forma, a pesquisa realizada.

\section{AGRADECIMENTOS}

Ao CNPq/CAPES pelo suporte financeiro. Ao Laboratório de Referência em Dessalinização (LABDES) do Centro de Ciências e Tecnologia da UFCG, pelo uso de suas instalações e apoio à pesquisa.

\section{BIBLIOGRAFIA}

[1] MENEZES, R. R., NEVES, G. A., FERREIRA, H. C., "O estado da arte sobre o uso de resíduos como matérias-primas cerâmicas alternativas”, Revista Brasileira de Engenharia Agrícola e Ambiental, v. 6, n. 2, pp. 303-313, 2002.

[2] MENEZES, R. R., NEVES, G. A., FERREIRA, H. C., LIRA, H. L., "Recycling of granite industry waste from the northeast region of Brazil”, Environmental Management and Health, West Yorkshire, v. 13, n.2, pp. 134-145, 2002.

[3] CORREIA, J. R., BRITO, J., PEREIRA, A. S., "Effectson concrete durability of using recycled ceramic aggregates", Materials and Structures, v. 39, n. 2, pp.169-177, 2006. 
[4] ANGUlO, S. C., TEXEIRA, C. E., CASTRO, A. L., NOGUEIRA, T. P., "Resíduos de construção e demolição: avaliação de métodos de quantificação", Revista Engenharia Sanitária e ambiental, v. 16, n. 3, pp. 299-306, 2011.

[5] BENITO, J.M., CONESA, A., RUBIO, F., RODRÍGUEZ, M.A., "Preparation and characterization of tubular ceramic membranes for treatment of oil emulsions", Journal of the European Ceramic Society, v. 25, n. 4, pp. 1895-1903, 2005.

[6] SILVA, F. A., LIRA, H. L., "Preparação e caracterização de membranas cerâmicas de cordierita", Ceramica, v. 52, n. 324, pp. 276-282, 2006.

[7] LIMA, R. C. O., LIRA, H. L., NEVES, G. A., et al., "Aproveitamento do resíduo de serragem de granito para fabricação de membranas cerâmicas de baixo custo", Revista Eletrônica de Materiais e Processos, v.6, n.3, pp. 163-169, 2011.

[8] NBR-6459/ABNT - Determinação do Limite de Liquidez de Solos.

[9] NBR-7180/ABNT - Determinação do Limite de Plasticidade de Solos.

[10] MACEDO, R. S., MENEZES, R. R., NEVES, G. A., FERREIRA, H. C., "Estudo de argilas usadas em cerâmica vermelha", Cerâmica, v. 54, n. 332, pp. 411-417, 2008.

[11] CAPUTO, H. P., Mecânica dos solos e suas aplicações fundamentais, $6^{\circ}$ ed., Rio de Janeiro, LTC Livros técnicos e científicos editora S.A, 1988.

[12] CHAVES, A. C., LIRA, H. L., NEVES, G. A., et al., "Obtenção e caracterização de membranas cerâmicas tubulares a partir de massas incorporadas com argila, caulim e quartzo", Cerâmica, v. 59, n. 349, pp. 192197, 2013.

[13] DHANAPANDIAN, S., MANOHARAN, C., SUTHARSAN, P, "Influence of firing temperature on alluvial clay brick properties in india", International Journal of Current Physical Sciences, v. 1, n.4, pp. 14$18,2011$.

[14] AMADO, J. D. S., VILLAFRADES, P. Y. M., TUTA, E. M. C., "Caracterización de arcillas y preparación de pastas cerámicas para la fabricación de tejas y ladrillos em la región de barichara, Santander", Dyna, v. 78, n.167, pp. 53- 61, 2011.

[15] SILVA, M. C., OLIVEIRA, R. C., LIRA, H. L., et al., "Obtenção de membrana cerâmica para tratamento de efluente da indústria têxtil", Revista Eletrônica de Materiais e Processos, v.9, n. 2, pp. 81-85, 2014.

[16] SILVA, M. C., LIRA, D. S., FREITAS, N. L., et al., "Membranas cerâmicas assimétricas tubulares: influência do tempo de deposição da camada filtrante na morfologia das membranas", Matéria (Rio J.), v.20, n.2, pp.335-343, 2015. 